\title{
Long-COVID postural tachycardia syndrome: an American Autonomic Society statement
}

\author{
Satish R. Raj ${ }^{1,2}$ ( Amy C. Arnold ${ }^{2,3} \cdot$ Alexandru Barboi $^{4} \cdot$ Victoria E. Claydon $^{5} \cdot$ Jacqueline K. Limberg $^{6} \cdot$ \\ Vera-Ellen M. Lucci ${ }^{5}$. Mohammed Numan ${ }^{7}$. Amanda Peltier ${ }^{8} \cdot$ Howard Snapper $^{9} \cdot$ Steven Vernino ${ }^{10}$ on behalf of the \\ American Autonomic Society
}

Received: 20 February 2021 / Accepted: 10 March 2021 / Published online: 19 March 2021

(c) Springer-Verlag GmbH Germany, part of Springer Nature 2021

\begin{abstract}
COVID-19 is a global pandemic that has had a devastating effect on the health and economy of much of human civilization. While the acute impacts of COVID-19 were the initial focus of concern, it is becoming clear that in the wake of COVID19, many patients are developing chronic symptoms that have been called Long-COVID. Some of the symptoms and signs include those of postural tachycardia syndrome (POTS). Understanding and managing long-COVID POTS will require a significant infusion of health care resources and a significant additional research investment. In this document from the American Autonomic Society, we outline the scope of the problem, and the resources and research needed to properly address the impact of Long-COVID POTS.
\end{abstract}

Keywords COVID $\cdot$ Long COVID $\cdot$ Postural tachycardia syndrome $\cdot$ Position statement

\section{Statement of the problem}

The novel coronavirus (SARS-CoV-2) emerged in Wuhan, China, in late 2019. It quickly became a pandemic, significantly impacting health and the economy worldwide $[1,2]$. As of March 8, 2021, there have been over 116,000,000 cases of COVID-19 (the disease caused by SARS-CoV-2), and almost 2,600,000 deaths attributed to COVID-19 worldwide [3]. This health care crisis has imposed an unprecedented strain on society in general, and in particular has challenged the ability of health care organizations to provide

Satish R. Raj

satish.raj@ucalgary.ca

1 Department of Cardiac Sciences, Libin Cardiovascular Institute, University of Calgary, GAC70 HRIC Bldg, 3280 Hospital Dr. NW, Calgary, AB T2N 4Z6, Canada

2 Division of Clinical Pharmacology, Department of Medicine, Autonomic Dysfunction Center, Vanderbilt University Medical Center, Nashville, TN, USA

3 Department of Neural and Behavioral Sciences, Pennsylvania State University College of Medicine, Hershey, PA, USA

4 Department of Neurology, NorthShore University Health System, Pritzker School of Medicine, University of Chicago, Chicago, IL, USA adequate care, including to non-COVID-19 patients. While the acute illness has rightly been the initial focus of medical attention, it is now clear that some patients are left with a constellation of ongoing symptoms even after resolution of the acute illness. These ongoing symptoms have been termed Long-COVID, "post-acute COVID syndrome" (PACS), "post-acute sequelae of SARS-CoV-2 syndrome" (PASC), "post-COVID syndrome" or "long-haul COVID". The purpose of this statement from the American Autonomic Society is to provide a statement of need to guide physicians,

5 Department of Biomedical Physiology and Kinesiology, Simon Fraser University, Burnaby, BC, Canada

6 Department of Nutrition and Exercise Physiology, University of Missouri, Columbia, MO, USA

7 Division of Cardiology, Department of Pediatrics, University of Texas Health Sciences Center at Houston, Houston, TX, USA

8 Department of Neurology, Autonomic Dysfunction Center, Vanderbilt University Medical Center, Nashville, TN, USA

9 Cardiology Division, Wellstar Healthcare System, Atlanta, GA, USA

10 Department of Neurology, UT Southwestern Medical Center, Dallas, TX, USA 
health systems, researchers and research funding agencies about the impact of Long-COVID POTS.

The United Kingdom National Institute for Health and Care Excellence (NICE) [4] has defined various symptomatic phases of COVID-19. These include:

- "Acute COVID-19" which includes signs and symptoms up to 4 weeks following onset of illness

- "Ongoing symptomatic COVID-19" for signs and symptoms of COVID-19 from 4 to 12 weeks after the onset of illness

- "Post-COVID-19 syndrome" for signs and symptoms that develop during or after an infection consistent with COVID-19, continue for $>12$ weeks and are not explained by an alternative diagnosis

- "Long-COVID" that includes both ongoing symptomatic COVID-19 (from 4 to 12 weeks) and post-COVID-19 syndrome (12 weeks or more).

\section{Long-COVID POTS}

Commonly described symptoms of Long-COVID include some combination of breathlessness, palpitation, chest discomfort, fatigue, pain, cognitive impairment ("brain fog”), sleep disturbance, orthostatic intolerance, peripheral neuropathy symptoms (pins and needles, and numbness), abdominal discomfort, nausea, diarrhea, joint and muscle pains, symptoms of anxiety or depression, skin rashes, sore throat, headache, earache and tinnitus $[4,5]$. These symptoms, when combined with excessive orthostatic tachycardia, can lead to a diagnosis of postural tachycardia syndrome (POTS) post-COVID-19 [6]. Recently, a number of case reports have been published describing patients who developed POTS following a SARS-CoV-2 infection [7-9]. Anecdotal preliminary information from personal communications with autonomic clinics in the USA, UK and Canada suggest an increased incidence of POTS, with independent articles in the Wall Street Journal [10], The New York Times [11], and The Atlantic [12]. The diagnosis of POTS requires excessive orthostatic tachycardia (heart rate increase of $>30$ beats per minutes in adults [ $>40$ beats per minute in patients age 12-19 years] within 10 minutes of assuming upright posture) in the absence of orthostatic hypotension, with associated symptoms of orthostatic intolerance, for at least 3 months [13]. Therefore, if related to COVID-19, these patients would be in the "post-COVID-19 syndrome" window (>12 weeks post-infection) and termed "Long-COVID POTS".

\section{Health system impacts of Long-COVID POTS}

The number of physicians familiar with the care of POTS is insufficient for the existing (pre-COVID-19) patient volume. The waiting lists in tertiary care clinics with this expertise can be 6-12 months or longer, leading to long delays in diagnosis and treatment [14]. Multidisciplinary integrated clinical care is rarely available, even though symptoms in these patients cross over into multiple specialties.

Anecdotal experience reported in personal communications (Dr. Tae H Chung from Johns Hopkins University School of Medicine and Dr. Brent P Goodman from the Mayo Clinic in Arizona) indicates an increase in the number of patients referred to autonomic specialty clinics, and this is likely to increase as the full impacts of COVID-19 are realized. In order to properly care for these patients, more clinical resources will be needed. This will require increased infrastructure to improve throughput in the current secondary and tertiary care referral clinics, including staff and testing capacity. In order to effectively handle this expected increased patient load, providers may have to start caring for these patients outside of the referral centers. Autonomic experts should share their expertise and management protocols to help patients and other providers, recognizing that this might evolve as knowledge about these conditions increases. These patients have complicated medical conditions that will require longer visits and more allied health care personnel (including nurses, physiotherapists and psychologists) to deliver the needed care. In other words, it will not be possible to address the needs of this population without a commitment not just from the providers, but also hospitals and medical center administration.

\section{Research opportunities to understand Long-COVID POTS}

There is much that is not known about Long-COVID POTS. While not exhaustive, the list includes:

1. Natural history of Long-COVID POTS. Is this a selflimiting disorder that will resolve in several months, or one that will last for years?

2. Underlying pathophysiology of Long-COVID POTS. Are the symptoms related to specific inflammatory pathways or autoimmunity? Is low blood volume a feature? The renin-angiotensin-aldosterone system, which is critical in blood volume regulation, has been implicated in the acute SARS-CoV-2 viral penetration of human cells [15, 
16]. Could there be renal impairment? Is cerebral blood flow affected by SARS-CoV-2 [17]? Could hypoxia from residual lung damage be present and contribute to cognitive impairment? Does a sensory or autonomic neuropathy develop following the acute illness? In addition, there is also increased incidence of acute motor and sensory axonal neuropathy (AMSAN) and acute demyelinating inflammatory demyelinating polyneuropathy (AIDP) associated with COVID-19, both of which may have autonomic dysfunction as a component $[18,19]$. Long-COVID POTS may also represent a version of acute pan-dysautonomia which is typically post-infectious autonomic neuropathy similar to AIDP and AMSAN.

3. Are patients with Long-COVID POTS different from patients with POTS unrelated to COVID-19? While POTS is a heterogeneous condition, there is a significant subgroup of patients who have reported their symptom onset to occur shortly after a viral illness [14]. This raises the question as to whether the sudden increase in the clinical presentation of post-COVID-19 POTS is a phenomenon specific to the SARS-CoV-2 virus and COVID-19, or a more general post-viral response. In the latter case, the connection between POTS and COVID19 would be because SARS-CoV-2 is currently a commonly diagnosed virus, and not because of a unique mechanism of COVID-19 causing POTS. Do these patients have objective evidence of abnormalities on autonomic testing? Other objective pathophysiological abnormalities? Clarifying this question will have significant implications for defining the optimal treatments for these patients.

4. Best treatments for Long-COVID POTS. We need to rapidly engage in studies to learn how to best treat these patients. Could exercise re-training be beneficial as it can be for some POTS patients [20, 21]? It may be that different subpopulations require different targeted treatments, although this remains to be confirmed.

\section{Research investments needed to understand Long-COVID POTS}

Federal funding initiatives designed to support a deeper comprehensive understanding of POTS are needed, in addition to the intentional investment in research to understand Long-COVID POTS [22]. In addition to the National Institutes of Health initiative and the Medical Research Council in the United Kingdom, we advocate for similar increases in research funding from the National Health and Medical Research Council in Australia, the Canadian Institutes of Health Research in Canada, and other leading research agencies worldwide.

\section{Conclusions}

COVID-19 has had a massive acute effect on the world's health and economy. While most people with COVID-19 illness recover completely, others continue to experience chronic and diverse symptoms including autonomic manifestations. Addressing the patient needs of Long-COVID, and especially Long-COVID POTS, will take a significant investment of resources and funding, both for clinical care and research. It is imperative to act during this window of opportunity in hopes of reducing or shortening the burden of symptoms for these patients.

Funding This work was supported in part by the National Institutes of Health Grant UL1 TR000445 and R00 HL122507.

\section{Declarations}

Conflict of interest SRR has grant support from the Canadian Institutes of Health Research (Ottawa, Canada) and Dysautonomia International (East Moriches, NY, USA). SRR is a consultant for Lundbeck LLC and Theravance Biopharma, and serves as DSMB chair for a phase 2 study run by Arena Pharmaceuticals. SRR has received compensation for editorial activities (Associate Editor) with Autonomic Neuroscience-Basic and Clinical. ACA: Consultant for National Vaccine Injury Compensation Program, U.S. Department of Health and Human Services; research grant from Dysautonomia International. AB: None. VEC: Research grants from the Heart and Stroke Foundation of Canada, Craig H Nielsen Foundation, and International Collaboration on Repair Discoveries. JKL: None. MN: None. VEML: None. AP: None. HS: None. SV: SV has received research support from Biohaven, Dysautonomia International, Genentech, Grifols, Rex Griswold Foundation, and Athena/Quest Diagnostics (through a licensing contract) and personal compensation for consulting for Catalyst, Sage and Alterity.

\section{References}

1. Centers for Disease Control and Prevention (2021) COVID-19 Cases, Data and Surveillance. https://www.cdc.gov/coronavirus/ 2019-ncov/cases-updates/. Accessed 16 Feb 2021

2. World Health Organization (2021) Coronavirus disease (COVID-19) weekly epidemiological update and weekly operational update. https://www.who.int/emergencies/diseases/novelcoronavirus-2019/situation-reports/. Accessed 16 Feb 2021

3. World Health Organization (2021) WHO coronavirus (COVID19) dashboard. https://covid19.who.int/. Accessed 8 Mar 2021

4. National Institute for Health and Care Excellence (NICE) (2021) COVID-19 rapid guideline: managing the long-term effects of COVID-19. https://www.nice.org.uk/guidance/ng188. Accessed 16 Feb 2021

5. Mayo Clinic (2021) COVID-19 (coronavirus): long-term effects. https://www.mayoclinic.org/coronavirus-long-term-effects/art20490351. Accessed 20 Feb 2021

6. Goldstein DS (2020) The possible association between COVID19 and postural orthostatic tachycardia syndrome. Heart Rhythm. https://doi.org/10.1016/j.hrthm.2020.12.007 
7. Kanjwal K, Jamal S, Kichloo A, Grubb BP (2020) New-onset postural orthostatic tachycardia syndrome following coronavirus disease 2019 infection. J Innov Card Rhythm Manag 11:4302-4304

8. Miglis MG, Prieto T, Shaik R et al (2020) A case report of postural tachycardia syndrome after COVID-19. Clin Auton Res 30:449-451

9. Umapathi T, Poh MQW, Fan BE et al (2020) Acute hyperhidrosis and postural tachycardia in a COVID-19 patient. Clin Auton Res 30:571-573

10. Reddy S (2020) For COVID long-haulers, a little-known diagnosis offers possible treatments-and new challenges. Wall Street Journal. https://www.wsj.com/articles/for-covid-long-haulers-alittle-known-diagnosis-offers-possible-treatmentsand-new-chall enges-11606761541

11. Gulliver Caspersen S (2020) When the doctor is a COVID 'long hauler.' New York Times. https://www.nytimes.com/2020/10/21/ well/live/when-the-doctor-is-a-covid-long-hauler.html

12. Yong E (2020) Long-Haulers Are redefining COVID-19. The Atlantic. https://www.theatlantic.com/health/archive/2020/08/ long-haulers-covid-19-recognition-support-groups-symptoms/ 615382/

13. Raj SR, Guzman JC, Harvey P et al (2020) Canadian cardiovascular society position statement on postural orthostatic tachycardia syndrome (POTS) and related disorders of chronic orthostatic intolerance. Can J Cardiol 36:357-372. https://doi.org/10.1016/j. cjca.2019.12.024

14. Shaw BH, Stiles LE, Bourne K et al (2019) The face of postural tachycardia syndrome-insights from a large cross-sectional online community-based survey. J Intern Med 286:438-448. https://doi.org/10.1111/joim.12895
15. Shang J, Wan Y, Luo C et al (2020) Cell entry mechanisms of SARS-CoV-2. Proc Natl Acad Sci 117:11727-11734. https://doi. org/10.1073/pnas.2003138117

16. Hoffmann M, Kleine-Weber H, Schroeder S et al (2020) SARSCoV-2 cell entry depends on ACE2 and TMPRSS2 and is blocked by a clinically proven protease inhibitor. Cell 181:271-280.e8. https://doi.org/10.1016/j.cell.2020.02.052

17. Fraiman P, Freire M, Moreira-Neto M, Godeiro-Junior C (2020) Hemorrhagic stroke and COVID-19 infection: coincidence or causality? NeurologicalSci 21:100274

18. Filosto M, Cotti Piccinelli S, Gazzina S et al (2020) GuillainBarré syndrome and COVID-19: an observational multicentre study from two Italian hotspot regions. J Neurol Neurosurg Psychiatry. https://doi.org/10.1136/jnnp-2020-324837

19. Arcila-Londono X, Lewis RA (2012) Guillain-Barré syndrome. Semin Neurol 32:179-186. https://doi.org/10.1055/s-0032-13291 96

20. Fu Q, Vangundy TB, Galbreath MM et al (2010) Cardiac origins of the postural orthostatic tachycardia syndrome. J Am Coll Cardiol 55:2858-2868. https://doi.org/10.1016/j.jacc.2010.02.043

21. Gibbons CH, Silva G, Freeman R (2021) Cardiovascular exercise as a treatment of postural orthostatic tachycardia syndrome: a pragmatic treatment trial. Heart Rhythm. https://doi.org/10.1016/j. hrthm.2021.01.017

22. National Institutes of Health Notice of Intent to Publish Research Opportunity Announcements (OTA-21-015) for the Post-Acute Sequelae of SARS-CoV-2 Infection (PASC) Initiative. https:// covid19.nih.gov/sites/default/files/2021-02/PASC-Pre-Notice. pdf. Accessed 20 Feb 2021 